\title{
The Acute Compartment Syndrome of the Lower Leg: A Difficult Diagnosis?
}

\author{
P.P. Oprel ${ }^{1}$, M.G. Eversdijk ${ }^{1}$, J.Vlot ${ }^{2}$, W.E. Tuinebreijer ${ }^{1}$ and D. den $\operatorname{Hartog}^{*}, 1$ \\ Department of ${ }^{I}$ Surgery-Traumatology and ${ }^{2}$ Pediatric Surgery, Erasmus MC, University Medical Center Rotterdam, \\ P.O. Box 2040, 3000 CA Rotterdam, The Netherlands
}

\begin{abstract}
Three patients, two adults and one child, developed an acute compartment syndrome of the lower leg. Due to delay in diagnosis, severe complications developed, resulting in two transfemoral amputations. In the youngest patient, the lower leg was able to be saved after extensive reconstructive surgery. In most cases, acute compartment syndrome of the lower leg is seen in combination with a fracture (40\%), although other causes (minor trauma or vascular surgery) are also known. Moreover, patient history (pain out of proportion to the associated injury) and physical examination are central to the diagnosis. In some cases, however, a reliable diagnosis cannot be made clinically, as in the case of unconscious, intoxicated or intubated patients, as well as small children. Under these circumstances, intra-compartmental pressure measurement can be of great assistance. After confirmation of the diagnosis, immediate fasciotomy of all lower leg compartments should be performed. The eventual outcome of this syndrome is directly related to the time elapsed between diagnosis and definitive treatment. Although the diagnosis can be difficult, delays in treatment should be avoided at all costs. The acute compartment syndrome of the lower leg is a surgical emergency and should be dealt with immediately.
\end{abstract}

Keywords: Compartment syndrome, bone fracture, leg.

Acute compartment syndrome (ACS) of the lower leg that is not recognized and left untreated can lead to loss of the affected extremity. In some cases, the ACS can result in a life-threatening condition. Prognosis for full recovery is strongly dependent on an early diagnosis and treatment. Postponing treatment because of late recognition and additional time-consuming diagnostic procedures increases the risk of complications. The timely recognition of ACS of the lower leg can be difficult because it can be caused by minimal trauma or masked by a concomitant trauma $[1,2]$.

No gold standard exists for diagnosing ACS. The amount of pain experienced and, particularly, the discrepancy between the seriousness of pain in comparison with the extent of trauma can be an indication for the existence or the development of an ACS. Patient history and physical examination are very important in making the diagnosis. In some cases, intra-compartmental pressure measurement can facilitate diagnosis.

In stark contrast to the controversy surrounding diagnosis, more agreement exists about the treatment of ACS. There is a consensus that opening the four compartments of the lower leg as wide as possible and decompressing is the only method adequate to treat ACS [3]. Early treatment is mandatory in order to achieve a good result.

*Address correspondence to this author at the Department of SurgeryTraumatology, Erasmus MC, University Medical Center Rotterdam, 's Gravendijkwal 230, Office H-960, 3015 CE Rotterdam, The Netherlands; Tel: +31 10 7032395; Fax: +31 10 7032396;

E-mail: d.denhartog@erasmusmc.nl
In this publication, we describe three patients with a complicated course of ACS of the lower leg after trauma. We also give a review of the diagnostic possibilities and suggest guidelines to recognise the compartment syndrome in its early phases.

Patient A, a healthy 28-year-old female, was seen at the Emergency Department of a regional hospital after an accident with her scooter; she hit a post with her lower leg. She was treated according to the principles of the Advanced Trauma Life Support (ATLS). The patient had only sustained a comminuted fracture of the left tibial plateau (Fig. 1). A CT-scan of the left knee was performed and the joint was temporarily immobilised with an upper plaster splint. The patient was hospitalised. One and a half days after the accident, motor function was absent in the lower left leg and absence of sensation in the foot was noted. ACS was diagnosed and a four compartment fasciotomy was performed to decompress the lower leg compartments. The patient was transferred to a central hospital for further treatment of the tibial fracture. Upon inspection under anaesthesia (for particular evaluation of muscle vitality) four days after trauma, the muscle tissue of the anterior, peroneal and deep flexor compartments was not vital. Of the superficial flexor compartment, only the medial muscle belly of the gastrocnemius muscle was still vital. After consultation with the patient, it was decided that in view of the prognosis associated with absent sensation and motor function in the lower leg and the fact that she became seriously ill because of tissue necrosis, the appropriate course of action was performance of an upper amputation. Seven days after the accident, a transfemoral amputation was carried out. Next the patient underwent outpatient rehabilitation with an upper leg prosthesis. 


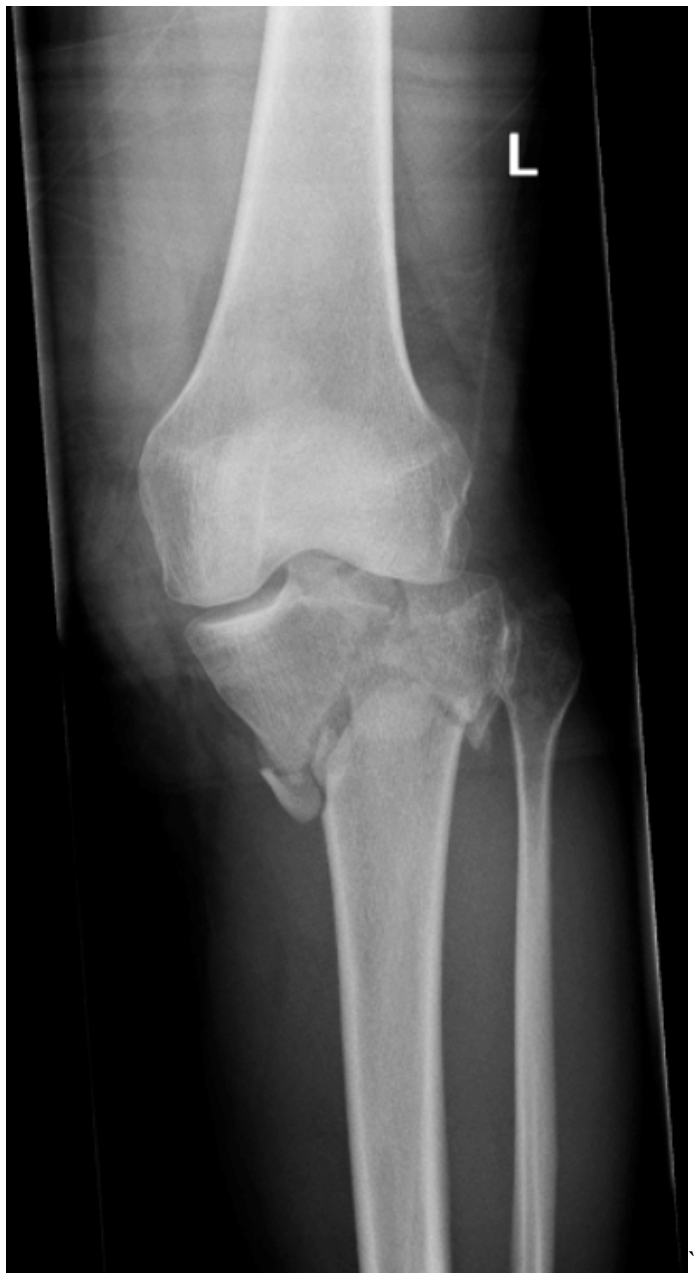

Fig. (1). Comminuted left tibial plateau fracture (Schatzker V) of patient A.

Patient B, a healthy 30-year-old man, sustained a right proximal crural fracture as a result of a direct trauma during a soccer match (Fig. 2). For this, he was admitted to a regional hospital and operated on the same day. The proximal fracture of the right lower leg was stabilised with an intramedullary nail (Fig. 3). The next day, the patient developed an ACS of the right lower leg, for which a four compartment fasciotomy was performed with a medial and lateral incision. The postoperative course was complicated by increasing pain symptoms of the right lower leg, as well as high fever. Because a wound infection was suspected, a course of intravenous antibiotics was begun. The patient was followed up with MRI-scans to rule out a deep infection or osteomeyelitis. On these scans, a collection of fluid was seen; therefore, an exploration and drainage of the wound was carried out. The wound was treated with a vacuum system. Due to a lack of healing, the patient was transferred after one month to a central hospital for a second opinion. At the first outpatient visit, the patient still experienced much pain in the proximal lower leg. Sensation was almost completely absent in the foot and the lateral lower leg. No extension or flexion of the foot or toes was possible. Upon wound inspection, free exposed bone was found. Neurological examination showed complete loss of the peroneal and tibial nerves. It was decided to admit the patient for wound exploration under general anaesthesia in the operation room. Necrosis of the anterior, peroneal and deep flexor compartments was found. Only the superficial flexor compartment (proximal area) showed some vital muscle tissue. After consultation with the patient and his partner, it was decided to perform a transfemoral amputation. The postoperative course was marked by serious coping problems. This patient also started upon an outpatient rehabilitation course with upper leg prosthesis.

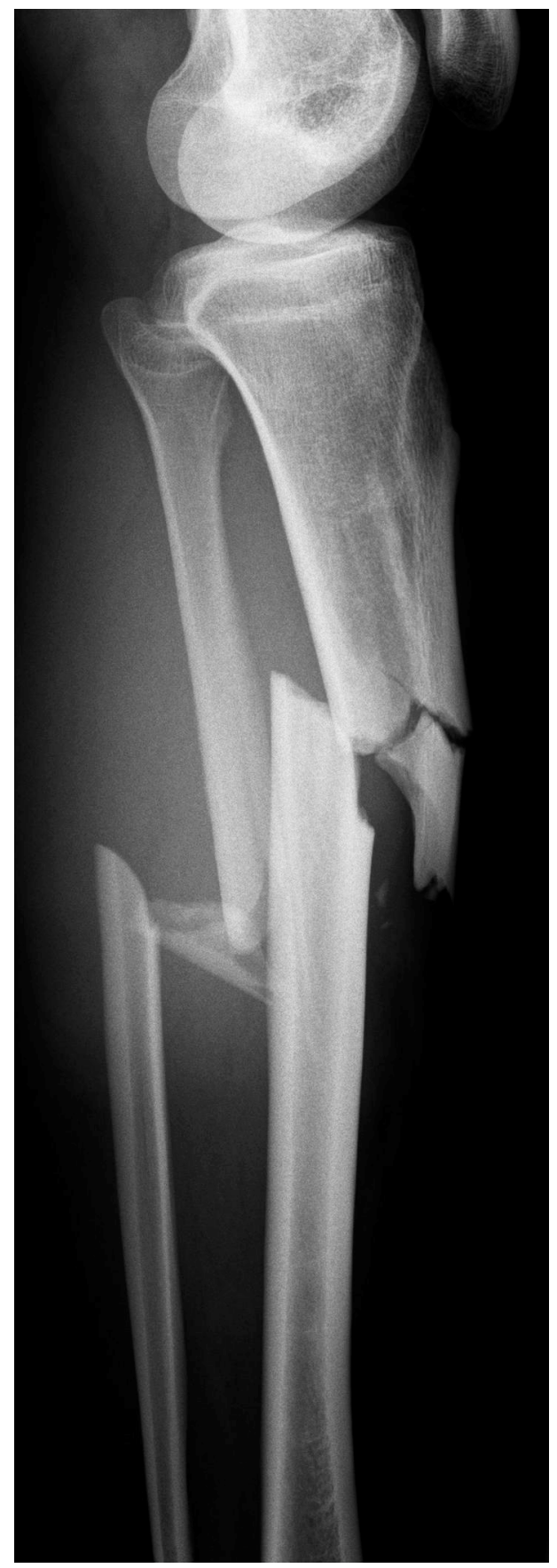

Fig. (2). Proximal right lower leg fracture of patient B.

Patient C, a healthy four-year-old boy, was hit by a car driving at a speed of $40 \mathrm{~km} /$ hour while he was walking. The abdomen and right lower leg were very painful. He was admitted to a regional hospital where a right tibial fracture, 


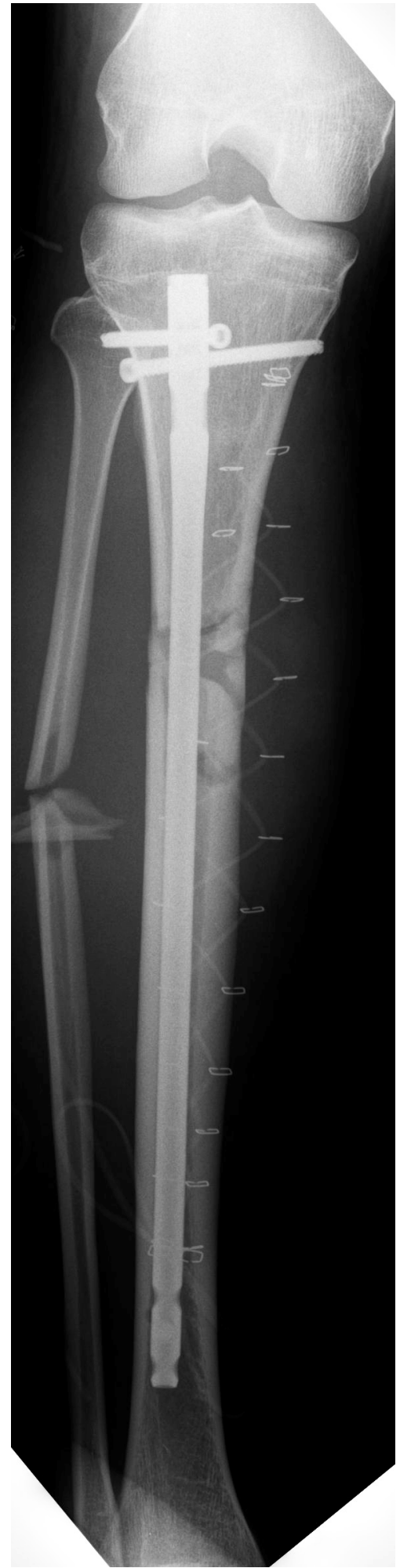

Fig. (3). Situation of patient B after intramedullary nail fixation and fasciotomy of the right lower leg.

right subcapital humeral fracture and free intra-abdominal fluid were diagnosed. Upon laparotomy, a liver rupture, which needed packing, was observed. During the same session, a head wound was treated. The right lower leg stood in an abnormal position, but the foot exhibited normal circulation. After reposition the tibial fracture was treated with an upper plaster cast. The same day, the patient was intubated and ventilated, then transferred to a central hospital for further treatment. At inspection on the second day after the trauma, diffuse oedema of the lower leg was seen. On the same day, gauze around the liver was removed at relaparotomy. On the third day, the toes of the right leg were pale and cold. After splitting the plaster, substantial capillary refill was observed. On the fourth day, the patient was extubated. Six days after trauma he complained of pain in the right leg. After removal of the plaster, extensive blistering and oedema of the lower leg with partially threatened skin was seen. The same day a four compartment fasciotomy of the right lower leg was performed. Inspection showed extensive necrosis of the four muscle compartments. After this, the patient was operated upon many times, which at last resulted in the clearance of all compartments; only the medial head of the gastrocnemius muscle remained vital. The fracture was treated with an external fixator (Fig. 4). Nearly four weeks after the accident, the considerable defect at the right lower leg was covered with a free vascularised musculus latissimus dorsi flap. An outpatient rehabilitation course followed.

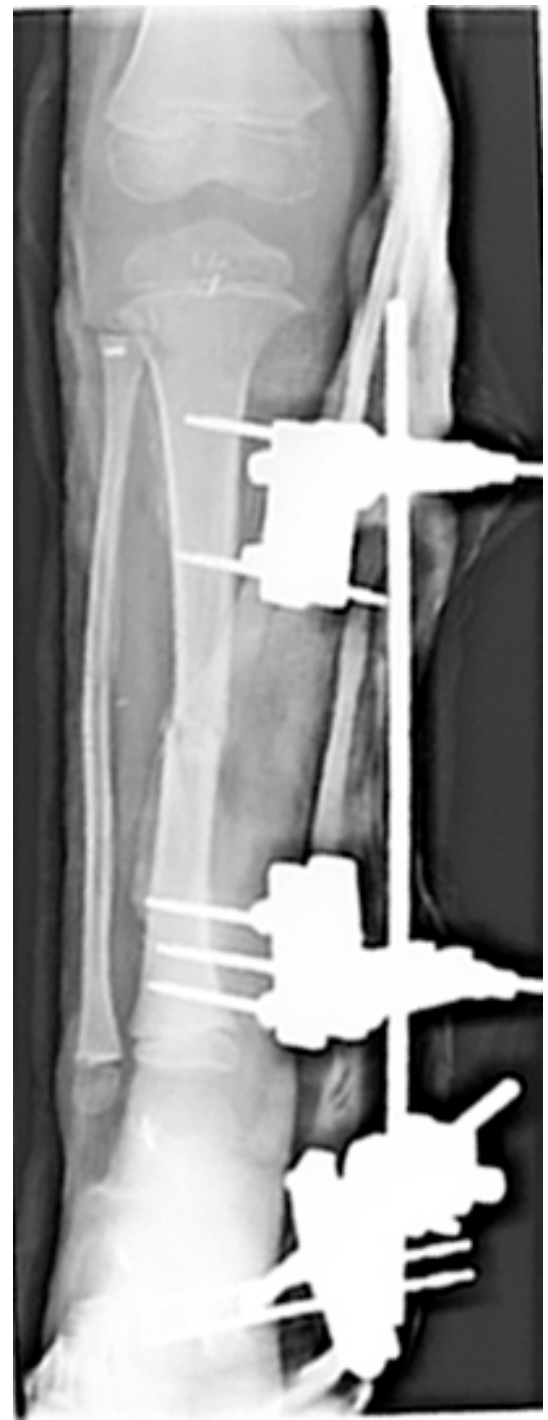

Fig. (4). Tibial fracture of patient $C$ treated with an external fixator.

The acute compartment syndrome of the lower leg usually arises after a direct trauma. In most cases, the ACS is 
seen with a fracture of the lower leg. About $40 \%$ of all compartment syndromes originate after a lower leg fracture $[1,4]$. Not only a tibial or crural fracture can cause an ACS, but also a tibial plateau fracture can give rise to an ACS (up to $30 \%$ with Schatzker type IV) [5]. In addition, an ACS can develop from intra-compartmental bleeding or as complication of a blunt trauma with contusion of the muscular tissue. Another reason can be vascular occlusion. Reperfusion oedema after vascular reconstruction or embolectomy leads to considerable pressure rise in the lower leg compartments, for relief of which fasciotomy may be necessary $[1,2]$.

Only the proximal part of the gastrocnemius muscle of patient $\mathrm{A}$ and $\mathrm{C}$ was not necrotic. This could be explained by the vascularisation of the heads of the gastrocnemius muscle, which is provided by the sural arteries and not the posterior tibial artery. The sural arteries are branches of the popliteal artery (Fig. 5). The posterior tibial artery vascularises the other muscles of the posterior compartment.

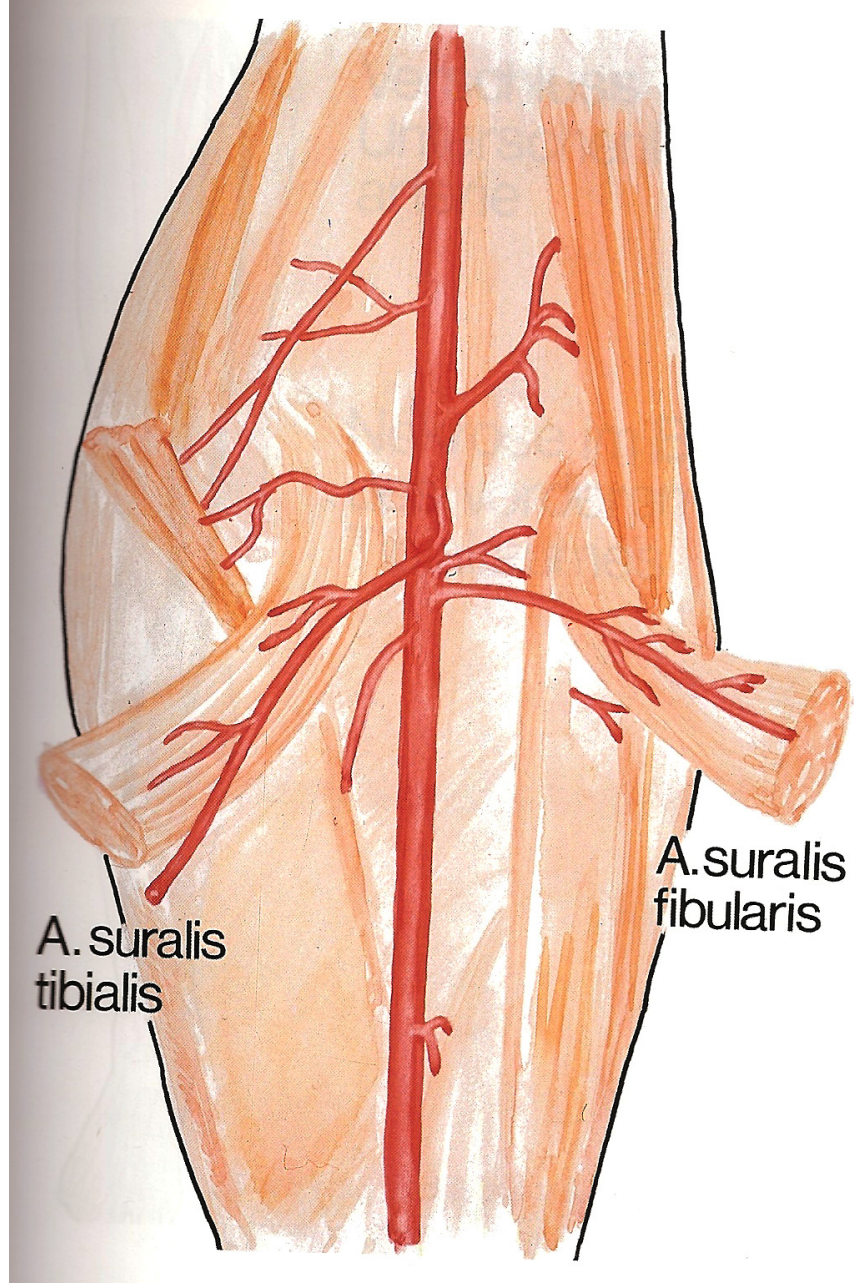

Fig. (5). The tibial and fibular sural arteries originate from the popliteal artery.

\section{PATIENT HISTORY}

A precise history of a patient who is suspected of having a compartment syndrome is very important. However, it is not always possible to obtain the history and sometimes the available history is not reliable. Examples of such cases are a comatose polytrauma patient, an intoxicated patient and young children. When information can be retrieved about the kind of injury, the possible existing discrepancy between the severity of the injury and the degree of pain, and the time course, these measures provide important support to the diagnosis.

\section{PHYSICAL EXAMINATION}

Upon inspection, the primary finding is swelling of the affected extremity. The inability to actively move flexors and extensors of the foot is an important indicator. Signs such as progression of pain complaints and pain with passive stretching of the affected muscles represent a warning of developing ACS. Furthermore, an applied plaster must be removed with progression of pain complaints in order to adequately assess the affected extremity. By recognizing these signs in a timely fashion, it is possible to identify an ACS early, so that the treatment can be started immediately.

Often a sensation disturbance in the web space between the first and second toes is found as a consequence of compression or ischemia of the deep peroneal nerve. This nerve is found in the anterior compartment; reduced sensibility represents a late sign of the ACS [6]. Because the intra-compartmental pressure seldom becomes higher than the arterial pressure, the absence of arterial pulsations is more often a sign of arterial injury than a very late sign of the ACS [2].

\section{DIAGNOSIS}

Patient history and physical examination play an important role in diagnosing ACS. In view of the sometimes variable time course of the ACS and its concurrence with other painful disorders, in some cases, history and physical examination are insufficient to arrive at a correct diagnosis. In these cases and in other situations where it is impossible to elicit a reliable history or to do a physical examination (lack of consciousness/coma, intoxication, little children, etc.), intra-compartmental pressure can offer a solution.

The normal pressure in a muscle compartment is between 10-12 $\mathrm{mm} \mathrm{Hg}$ [2]. It is, however, unclear how high measured pressure in the muscle compartment should be before committing to a fasciotomy. Mubarak et al. showed that an intra-compartmental pressure of $30 \mathrm{~mm} \mathrm{Hg}$ or more for 6-8 hours caused irreversible damage [7]. Generally, it is stated that an absolute pressure measurement of $30 \mathrm{~mm} \mathrm{Hg}$ or more in the compartment is a reason to do a fasciotomy [7].

Others relate the intra-compartmental pressure to the mean arterial pressure or to the diastolic pressure [8-10]. When the difference between the diastolic blood pressure and the measured intra-compartmental pressure is $30 \mathrm{~mm} \mathrm{Hg}$ or higher (the so- called "delta pressure"), this suggests ACS and is an indication for fasciotomy. Starting from this "delta pressure" instead of the absolute compartmental pressure prevents a number of unnecessary fasciotomies [8-10]. The highest pressure in a compartment was measured inside a zone localised $5 \mathrm{~cm}$ from the fracture. Outside this zone, the intra-compartmental pressure can fall $20 \mathrm{~mm} \mathrm{Hg}$; it is therefore advised to measure the pressure inside this zone and not in the fracture itself in order to prevent possible infectious complications [11]. 
When an ACS is present, research shows that in nearly all cases, the anterior compartment is affected. To diagnose ACS, it is therefore always necessary to measure the pressure in the anterior compartment; pressure measurements in the other compartments can be done upon indication [12].

\section{ANATOMY}

The lower leg has four compartments. These four compartments are bordered by the tibia, fibula, interosseous membrane and the surrounding fasciae. The anterior compartment contains the profound peroneal nerve, tibial anterior muscle, extensor hallucis longus and extensor digitorum muscle. In the lateral compartment are the nervus peroneus communis and superficial peroneal nerve, the peroneus longus and brevis muscle. In the deep posterior compartment are the arteria, vena and nervus tibialis, the posterior tibial muscle, musculus flexor hallucis longus and flexor digitorum longus. The superficial posterior compartment consists of the sural nerve, the gastrocnemius muscle and the soleus muscle.

\section{THERAPY}

Successful treatment of ACS requires surgical decompression as early as possible by way of a fourcompartment fasciotomy. Of course, the causes of external pressure also have to be removed, including a compressing bandage or plaster. An expectant position and (diagnostic) delays lead to serious complications. A direct relation exists between the time passed before the performance of a fasciotomy and the final functional result. An untreated compartment syndrome leads to loss of muscle tissue, hyperkalaemia, metabolic acidosis, renal insufficiency, amputation of extremities and sometimes death $[2,3,13$, 14].

The four compartments can be approached through one lateral incision or through a lateral and medial incision. It is important to make the skin incisions long enough (at least 16 $\mathrm{cm})$ so that all compartments are sufficiently decompressed $[7,15]$. The skin incision is left open so that later, after the swelling subsides, the wound can be closed.

A late diagnosis of ACS of the lower leg and the failure to perform an emergency fasciotomy can lead to the loss of an extremity. It is of the greatest possible importance to be alert for the symptoms that can point to the beginning of a compartment syndrome. This compartment syndrome can exist in children as well, as demonstrated by the cases formerly described. As early as possible, one has to think of the ACS, so that treatment may be begun as early as possible
[16]. The elapsed time between the onset of an ACS and treatment determines the ultimate result. Therefore, this period has to be as short as possible.

The case histories, which are described in this clinical lecture, show that ACS is sometimes difficult to diagnose and cases are for this reason rather often missed with serious consequences for the patient.

\section{REFERENCES}

[1] Elliott KG, Johnstone AJ. Diagnosing acute compartment syndrome. J Bone Joint Surg Br 2003; 85(5): 625-32.

[2] Gourgiotis S, Villias C, Germanos S, Foukas A, Ridolfini MP. Acute limb compartment syndrome: A review. J Surg Educ 2007; 64(3): 178-86.

[3] Rorabeck CH. The treatment of compartment syndromes of the leg. J Bone Joint Surg Br 1984; 66(1): 93-7.

[4] Ogunlusi JD, Oginni LM, Ikem IC. Compartmental pressure in adults with tibial fracture. Int Orthop 2005; 29(2): 130-3.

[5] Chang YH, Tu YK, Yeh WL, Hsu RW. Tibial plateau fracture with compartment syndrome: A complication of higher incidence in Taiwan. Chang Gung Med J 2000; 23(3): 149-55.

[6] Rorabeck CH, Macnab L. Anterior tibial-compartment syndrome complicating fractures of the shaft of the tibia. J Bone Joint Surg Am 1976; 58(4): 549-50.

[7] Mubarak SJ, Hargens AR, Owen CA, Garetto LP, Akeson WH. The wick catheter technique for measurement of intramuscular pressure. A new research and clinical tool. J Bone Joint Surg Am 1976; 58(7): 1016-20.

[8] McQueen MM, Court-Brown CM. Compartment monitoring in tibial fractures. The pressure threshold for decompression. J Bone Joint Surg Br 1996; 78(1): 99-104.

[9] Ovre S, Hvaal K, Holm I, Stromsoe K, Nordsletten L, Skjeldal S. Compartment pressure in nailed tibial fractures. A threshold of 30 $\mathrm{mmHg}$ for decompression gives $29 \%$ fasciotomies. Arch Orthop Trauma Surg 1998; 118(1-2): 29-31.

[10] Whitesides TE, Haney TC, Morimoto K, Harada H. Tissue pressure measurements as a determinant for the need of fasciotomy. Clin Orthop Relat Res 1975; 113: 43-51.

[11] Matava MJ, Whitesides TE, Jr., Seiler JG, III, Hewan-Lowe K Hutton WC. Determination of the compartment pressure threshold of muscle ischemia in a canine model. J Trauma 1994; 37(1): 50-8.

[12] McQueen MM, Christie J, Court-Brown CM. Acute compartment syndrome in tibial diaphyseal fractures. J Bone Joint Surg Br 1996; 78(1): 95-8.

[13] Finkelstein JA, Hunter GA, Hu RW. Lower limb compartment syndrome: Course after delayed fasciotomy. J Trauma 1996; 40(3): 342-4.

[14] Heemskerk J, Kitslaar P. Acute compartment syndrome of the lower leg: Retrospective study on prevalence, technique, and outcome of fasciotomies. World J Surg 2003; 27(6): 744-7.

[15] Mubarak SJ, Owen CA. Double-incision fasciotomy of the leg for decompression in compartment syndromes. J Bone Joint Surg Am 1977; 59(2): 184-7.

[16] Verleisdonk EJ, van der WC. [Missed acute compartment syndrome of the lower leg]. Ned Tijdschr Geneeskd 2004; 148(45): $2205-9$. 